\title{
Crossing: The INPR Journal
}

Vol. 1 (2020): 84-95

DOI: $10.21428 / 8766 \mathrm{eb} 43 . c 9 f d b e i a$

\section{Ce qui est donné et ce qui est espéré dans l'expérience}

\author{
Jérôme de Gramont \\ L'institut catholique de Paris \\ jerome.de-gramont@orange.fr
}

Nous naissons pour ainsi dire provisoirement quelque part ; c'est peu à peu que nous composons en nous le lieu de notre origine, pour y naître après coup, et chaque jour plus définitivement.

Rilke, Lettres milanaises, Lettre du 23 janvier 1923, Paris, Plon, 1956, p. 28

Devant ce qui naît en Grèce au tournant des VIIe et VIe siècles avant Jésus-Christ, revenant sur ce commencement proprement inouï qui correspond à une toute nouvelle attitude vis-à-vis du monde, le vieil Husserl n'hésite pas à parler d'une « humanité nouvelle», tout en sachant comme nous qu'humanité et monde sont bien plus anciens encore que la philosophie : avant le premier philosophe (Platon, lui qui invente la langue de la philosophie), ou le tout premier (Thalès, à qui il aura suffi de cette parole simple : «tout est eau » pour porter jusqu'à la pensée l'étant dans sa totalité) il y a le premier homme (Adam, à qui il revient d'avoir donné un nom à tous les animaux). Mais que le monde ait lieu avant la pensée n'ôte rien à ce commencement qui est tout de pensée : la possibilité de porter l'être jusqu'à la pensée, et rendre ainsi intelligible la totalité de l'étant ${ }^{1}$. Ce projet n'est pas loin de trouver avec Hegel un premier achèvement - ce qu'il annonce à Iéna lorsqu'il

${ }^{1}$ Là où Adam ne donne de nom qu'aux animaux qu'il voit défiler devant lui. 
propose de déposer l'ancien nom de la philosophie, celui d'amour de la sagesse, pour qu'elle devienne science effective - et ce qu'il réalise en quelque façon à Berlin, en publiant l'ultime édition de l'Encyclopédie et en prononçant des leçons où rien n'est oublié de ce qui a trait à l'Esprit, c'est-à-dire en déployant le Discours de la Raison selon les dimensions cardinales du monde. Ce qui revient à donner raison à la formule de Derrida, parlant de la philosophie occidentale : « le hegelianisme n'est que ce langage lui-même prenant absolument possession de $\operatorname{soi}^{2}$ ». Ce qui depuis Platon relevait d'un projet se voit donc bel et bien accompli : porter jusqu'au concept la totalité de ce qui est, jusqu'au recouvrement parfait de ce qui est effectif et de ce qui est rationnel.

C'est pourtant encore le même projet qui se répète à l'aube du XXe siècle avec la naissance de la phénoménologie, pour que phénomène et logos viennent à leur « commune présence », l'être rendu tout entier présent dans sa phénoménalité comme mouvement de se donner à nous, et la pensée tout entière ramenée au mouvement d'aller aux choses, ou plutôt aux choses mêmes. Là où il y a cercle, de ce qui se donne et de ce qui vise, il est possible d'y entrer par un côté ou par un autre. L'interprétation historique à laquelle se livre Husserl, et que résume l'expression par ailleurs si problématique d'idéalisme transcendantal, porte l'accent sur le côté du logos - aucun passage ne montre mieux sans doute comment la phénoménologie historique se place dans la suite du projet métaphysique que ces quelques lignes du $\S 6$ de la Krisis : "Porter la raison latente à la compréhension de ses propres possibilités et ouvrir ainsi au regard la possibilité d'une métaphysique en tant que possibilité véritable, c'est là l'unique chemin qui mette en route l'immense travail de réalisation d'une métaphysique, autrement dit d'une philosophie universelle ${ }^{3}$. » Mais c'est aussi la génialité de Husserl que d'avoir ouvert la voie à d'autres interprétations possibles du projet phénoménologique où l'accent se porte sur le libre événement du phénomène qui se donne à nous, avant toute compréhension mais aussi en l'appelant - à partir donc de ce qui se donne dans l'événement et son épreuve, encore aveugle et muette, dans l'attente d'être porté jusqu'à sa compréhension et son expression, ce que traduit cette fois la célèbre formule du $\S 16$ des Méditations cartésiennes : «Au commencement il y a l'expérience pure et, pour ainsi dire, muette encore, qu'il s'agit d'abord d'amener à l'expression pure de son propre sens ${ }^{4}{ } »$.

\footnotetext{
${ }^{2}$ Jacques Derrida, L'Ecriture et la différence, Paris, Seuil, 1967, p. 176.

${ }^{3}$ Edmund Husserl, La Crise des sciences européennes et la phénoménologie transcendantale, trad. G. Granel, Paris, Gallimard, 1976, p. 20 sq.

${ }^{4}$ Edmund Husserl, Méditations cartésiennes, cité d'après Claude Romano, Les Repères éblouissants, Paris, PUF, 2019, p. 61 - voir les traductions des Méditations par Levinas-Peiffer, Vrin, 1947, p. 33, et par Marc de Launay, Paris, PUF, 1994, p. 83 sq. Formule célèbre et à laquelle Merleau-Ponty s'est spécialement attaché puisqu'on la retrouve au commencement et à la fin de son parcours, dans
} 


\section{JÉRÔME DE GRAMONT}

Attachons-nous à la seconde version de ce projet phénoménologique dans la mesure où elle révèle davantage les déplacements opérés par la répétition du projet proprement métaphysique. Là où il était question d'être et pensée, là où il était possible de lire toute l'histoire de la métaphysique comme une suite de variations sur la phrase de Parménide : "Le même est à la fois penser et être " phénoménologie déploie son travail de description et de compréhension depuis deux autres piliers : l'expérience et le sens. Chacun de ces deux concepts fondamentaux demanderait assurément de très longs éclaircissements, allant bien au-delà d'un simple travail de définition puisqu'ils engagent l'Idée même de phénoménologie sans cesse à penser et repenser. De manière très provisoire, trop incomplète, et pour ne pas rejeter à l'infini ce que nous nous proposons d'exposer dans ces pages, contentons-nous de deux indications tirées de la formule husserlienne des Méditations cartésiennes :

- L'expérience est bien ce qui a lieu au commencement, et la vocation nativement descriptive de la phénoménologie est à demeurer à jamais fidèle à ce qui nous est donné dans l'expérience, quitte à raturer des descriptions qui semblaient jusque-là définitives ${ }^{6}$ ou même à corriger les principes qui devaient commander ces descriptions ${ }^{7}$.

- Le sens est ce qui vient par nous, comme ce que l'expérience seule (l'expérience pure et pour ainsi dire muette encore) est impuissante à nous livrer, de quelque manière que nous interprétions ensuite l'articulation entre ce qui nous arrive dans l'expérience et cette donation de sens qui cherche à lui répondre. Ainsi pouvonsnous souscrire à l'affirmation d'Aristote selon laquelle si l'homme n'existait pas les étoiles n'en continueraient pas moins à briller dans le $\mathrm{ciel}^{8}$, à condition d'ajouter que sans nous la lumière des étoiles ne brillerait d'aucun éclat, c'est-à-dire d'aucune beauté, c'est-à-dire à condition d'ajouter cette autre affirmation, de Kant dans la

l'Avant-propos de la Phénoménologie de la perception (Paris, Gallimard, 1945, p. X) et dans Le Visible et l'invisible (Paris, Gallimard, 1964, p. 171).

${ }^{5}$ Selon une affirmation lumineuse de Heidegger dans son cours Qu'appelle-t-on penser ?, trad. Paris, PUF, 1959, p. 224.

${ }^{6}$ Comme Husserl lui-même l'annonce dans les Ideen I, à la fin du § 96 (voir trad. P. Ricœur, Paris, Gallimard, 1950, p. 334 ; et trad. J.-F. Lavigne, Paris, Gallimard, 2018, p. 296).

${ }^{7}$ Comme nous l'avons montré dans notre étude « La phénoménologie comme lutte avec l'ange » dans Au commencement. Parole, Regard, Affect (Paris, Cerf, 2013). C'est sous la contrainte des phénomènes que Husserl est amené à corriger le «principe des principes » du § 24 des Ideen I.

8 «Et cependant les astres, même si nous ne les avions jamais vus, n’en seraient pas moins, je pense, des substances éternelles, distinctes de celles que nous connaissons » (Aristote, Métaphysique Z 16, 1040 b 34 (trad. Tricot). 
Critique de la faculté de juger : «sans les hommes la création tout entière serait un simple désert inutile et sans but final ${ }^{9}$ ».

Voilà qui dit encore trop peu, mais à partir de quoi il est possible d'avancer en deux directions. Celle de l'expérience d'abord, qui livre sans hiérarchie tout ce qui nous arrive, le plus infime comme le plus bouleversant, le rêve dont les contours s'estompent au petit matin comme la première nuit passée avec l'être aimé, les nuages « les merveilleux nuages » qui glissent sur le bleu du ciel comme la vastitude du ciel. Tout ce qu'il y a nous vient ainsi pêle-mêle, sable et neige, pierre et arbres, hommes et étoiles, sans premier plan ni arrière-plan, ou plutôt sans autre ordre que la succession contingente de nos impressions au fil du plus et du moins proche. Les journaux d'écrivain, où le plus anodin côtoie les séismes les plus profonds à même de secouer une vie, témoignent de cette égalité de l'expérience où, initialement et de droit, tout ce qui arrive est doté d'une égale dignité. Non seulement «au commencement est l'expérience », comme expérience pure et réduite à soi, sans le mélange d'aucune interprétation qui superposerait langage et signification à son mutisme, quand le phénomène s'offre à nous dans les limites de la seule expérience pour ainsi dire, mais encore "l'expérience elle-même est l'autorité », ce à quoi nos discours doivent revenir sans cesse pour y puiser leur ressource, sans quoi ils ne font que rêver autour de l'être au lieu de le dire. Ce qui emporte deux conséquences : l'ouverture de l'expérience (au pluriel des possibles) et son exposition (au danger). Parce que l'autorité est liée au fait de l'expérience et non à son contenu, aucune expérience particulière ne peut en effacer une autre au motif qu'elle aurait plus de poids ou de valeur. Ici congé doit être résolument donné aux discours de consolation (théodicées) qui s'efforcent de mesurer ce qu'il y a de bien ou de mal dans le monde pour décider qu'une part de l'expérience l'emporte définitivement sur l'autre. Autrement dit il n'y a pas d'expériences possibles auxquelles il faudrait faire plus de droit qu'à d'autres. Le retour d'Ulysse à Ithaque ne compte pas davantage que les péripéties de l'Odyssée (Circé, Calypso, Polyphème, les sirènes...) au motif qu'il clôt de manière heureuse une traversée riche en périls. Ulysse n'est pas moins Ulysse au milieu des épreuves chantées par Homère que dans le repos qu'il trouve au dernier chant auprès de Pénélope. Ulysse est l'histoire entière d'Ulysse. L'expérience en ellemême est sans privilège. Dit encore autrement, avec Jean-Yves Lacoste, les descriptions du phénoménologue portent à égalité sur tout ce qui se présente :

«La phénoménologie husserlienne se conçoit comme un retour à toute chose et n'importe quelle chose et non pas comme l'élucidation d'Erlebnisse privilégiés : tous les phénomènes sont égaux et d'égale importance. Face à ce qui lui apparaît, la conscience est toujours égale à elle-même : le même moi, muni des mêmes aptitudes à l'expérience, fait indifféremment l'expérience du beau et du laid, du bien et du mal,

\footnotetext{
${ }^{9}$ Kant, Critique de la faculté de juger, § 86, trad. A. Philonenko, Paris, Vrin, 1979, p. 250.
} 


\section{JÉRÔME DE GRAMONT}

de l'angoisse donc et de l'être-en-paix (dont Husserl, faut-il le préciser, ne parle pas) $)^{10}$. "

Une expérience ne se réfute pas, pas même au motif qu'une autre expérience se substituerait à elle ${ }^{11}$, chacune étant douée de la même autorité, de sorte que vaudrait pour le concept d'expérience ce que Stanislas Breton écrivait du monde dans une traduction par ailleurs discutable du premier aphorisme du Tractatus de Wittgenstein : «Le monde est tout ce qui arrive en nous tombant dessus ${ }^{12}$. Et si l'expérience nous livre à égalité tout ce qui arrive, c'est bien parce qu'elle s'ouvre en amont à tous les possibles, sans la moindre hiérarchie entre une expérience de premier plan et une expérience d'arrière-plan ${ }^{13}$. Ce qui veut dire qu'elle s'expose à égalité au plus heureux et au plus dangereux, à ce qui nous soulève de joie comme à "l'insoutenable vérité du désastre ${ }^{14}$ ». (Quand souffle le vent du Danger et que l'expérience ne s'y dérobe pas, le mot de Maurice Blanchot prend toute sa force : « L'expérience elle-même est l'autorité, mais l'autorité s'expie ${ }^{15}$ »).

Second pôle de cette Idée de la phénoménologie, la question du sens introduit une inégalité foncière dans l'égalité de l'expérience, ce qui nous arrive se distribuant désormais selon la différence de l'important et de l'insignifiant, du léger et du lourd, de l'habitable et de l'inhabitable, ou de ce qui nous soulève de joie et ce qui nous accable, autrement dit selon la dualité du sens et du non-sens. Là où l'expérience est à prendre comme un tout, là où il faut faire droit à ce que Claude Romano thématise comme holisme de l'expérience ${ }^{16}$, le sens naît par différence et contraste. Ainsi là où justice est rendue met-elle fin à l'injustifiable du mal qui régnait auparavant. Ainsi le beau, quand il surgit et déchire la trame du visible, suspend-il notre regard quotidien sur le monde - regard maintenant capturé, emporté, ravi. D’autres travaux auront à montrer comment cet événement du sens n'a proprement lieu que dans notre réponse, de sorte que même là où il prend sa naissance hors de nous il est juste de

\footnotetext{
${ }^{10}$ Jean-Yves Lacoste, Etre en danger, Paris, Cerf, 2011, p. 196.

${ }^{\text {" }}$ Pour reprendre l'exemple de Jean-Yves Lacoste : « la bataille de Waterloo ne critique ni ne contredit la bataille d'Austerlitz » (ibid., p. 205).

${ }^{12}$ Stanislas Breton, L'autre et l'ailleurs, Paris, Descartes \& Cie, 1995, p. 147, et L'avenir du christianisme, Paris, Desclée de Brouwer, 1999, p. 99.

${ }^{13} \mathrm{Ou}$ sans autre différence qu'entre le plus proche et le plus lointain, partant entre le plus aisément visible et ce qui nous demande un effort - ce qui tient seulement au lieu que nous occupons à un moment donné dans l'espace.

${ }^{14}$ Louis-René des Forêts, Ostinato, Paris, Gallimard, 1997, p. 88.

${ }^{15}$ Mot que Blanchot prononce dans une conversation avec Georges Bataille, et que celui-ci rapporte dans L'expérience intérieure (CEuvres complètes, t. V, Paris, 1973, p. 19, 67, 120).

${ }^{16}$ Voir Claude Romano, Au cœur de la raison, la phénoménologie, chap. XVII et XVIII, Paris, Gallimard, 2010.
} 
dire qu'il ne vient que par nous ${ }^{17}$. Qu'il suffise pourtant de tenir ces deux affirmations : que l'expérience est ce qui a lieu sans cesse et dès toujours, mais que le sens est proprement ce qui doit naître - et à ce titre ce qu'il nous appartient d'accueillir ou de faire venir, de porter jusqu'à son expression pure et sa pleine présence. Aussi ne peut-il venir que comme une possibilité adossée à sa possibilité adverse, comme une présence qu'il faut arracher à une absence, ou comme une affirmation qui doit s'enlever sur fond de menace. C'est là avouer qu'il ne peut y avoir de sens que marquée au sceau d'une incontournable fragilité ${ }^{18}$, parce que venant au milieu d'une histoire où se mêlent sans cesse sens et non-sens ${ }^{19}$. Comme c'est là reconnaître que la possibilité d'un effondrement du sens, précisément parce que ne s'agit que d'une possibilité, constitue une menace qui jamais ne pourra être définitivement écartée.

A la question métaphysique «Pourquoi y a-t-il quelque chose et non pas plutôt rien ? », il est possible d'objecter que l'évidence du quelque chose a définitivement révoqué l'hypothèse devenue impossible du rien. Mais à la question « Pourquoi y at-il du sens et non pas plutôt du non-sens ? " Husserl savait qu'il n'y a pas d'autre réponse dans notre histoire qu'une téléologie rejetant à l'infini, donc hors de notre portée, le dernier mot de la raison. De là ce danger de non-sens, danger toujours présent, dont aucune affirmation du sens ne pourra nous délivrer une fois pour toutes. Ce à quoi l'expérience nous expose ainsi, nous avons alors pour double tâche de le décrire ${ }^{20}$ et de lui résister ${ }^{21}$. Des travaux récents de Jean-Yves Lacoste montrent quelques variantes de ce Danger (quand il porte respectivement sur l'être, la parole et la vérité) et comment mener à bien cette double tâche. Citons-les brièvement comme autant de programmes phénoménologiques alors qu'ils sont déjà bien plus.

L'être se met en danger en ce qu'il autorise l'oubli. La donation excède le donné. Le sort de la substance est lié à l'événement. L'être enfin ne nous est

\footnotetext{
${ }^{17}$ Ce qui est de bonne phénoménologie, car il n’y a de phénomène que par celui qui le reçoit. Ce qui est tout conforme au principe défendu par Levinas, Chrétien et Marion selon lequel l'appel n'est entendu que dans la réponse (voir Jean-Luc Marion, Etant donné, Paris, PUF, 1997, p. 396 et références). Et ce dont l'esthétique kantienne fournit un exemple éclatant : il n’y a de beauté de la nature que dans le jugement de goût.

${ }^{18}$ Sur ce concept, central chez Jean-Louis Chrétien, voir notre article «La fragilité comme existential », Rue Descartes n ${ }^{\circ} 94,2017 / 4$.

${ }^{19}$ C'est une des leçons de Jacques Derrida dans L'Ecriture et la différence « que toute histoire ne puisse être, en dernière instance, que l'histoire du sens » (p. 54 note), mais aussi une histoire où le sens est lié au non-sens, la raison à la folie, le logos de la métaphysique à son excès...

${ }^{20}$ D'une certaine manière cette tâche proprement phénoménologique est déjà le fait de l'expérience littéraire, telle du moins que la pensée Blanchot, et telle qu'il en vient à la formuler dans la maxime de L'Ecriture du désastre : «Veiller sur le sens absent » (Paris, Gallimard, 1980, p. 72).

${ }^{21} \mathrm{Si}$ une promesse est possible que soit mis fin à la menace du non-sens, alors cette promesse n'est pas la nôtre, mais relève de la théologie.
} 


\section{JÉRÔME DE GRAMONT}

jamais confié que dans le prisme des modes d'être. Tout mode d'être ou presque est en danger. Mais si la topologie du provisoire est une topologie de l'être-en-danger, l'hypothèse du définitif, et ce que nous recevons comme anticipation du définitif, nous permet de penser un au-delà du danger, comme une paix définitive nous permet de penser un au-delà définitif de l'angoisse et des menaces de néant. ${ }^{22}$

Après avoir décrit un possible triomphe de la parole dans l'expérience excessive de la "lecture divine", une tâche plus sobre nous incombe, élucider la fragilité essentielle à toute parole - le danger dont elle ne peut s'affranchir et la fragilité propre à la parole liturgique. L'élucidation permettra de suggérer qu'il y a une histoire de la parole, dont la période présente est celle du nihilisme. Elle nous forcera alors à demander s'il y a des paroles capables de survivre au nihilisme, ou même de le surmonter. ${ }^{23}$

Le vrai est en danger, et il l'est d'abord en son essence. (...) Le logos est en danger, la ratio elle-même est en danger lorsqu'elle est réduite à un exercice scientifique, et la tâche du logos, lorsqu'il s'est plié à toutes les exigences du "rationnel", est de prendre pied sur des terres dont la ratio seule ne peut prendre connaissance. Le logos nous habilite à plus que la logique. Son domaine est plus vaste que celui de la rationalité, en son acception moderne. Et pour nommer l'excès de l'une par l'autre, disons que le logos, lorsqu'il fait plus que raisonner, a pour besogne propre celle de la pensée. ${ }^{24}$

Tirons une leçon aussi simple dans son énoncé que délicate à réaliser, à savoir qu'il n'y a pas de description du sens qui puisse faire l'économie d'une considération du non-sens. Pensée tenue à cette dualité des possibles, mais inégalement : le sens, parce qu'il peut venir ou ne pas venir, est affaire d'espérance («Qu'il vienne, qu'il vienne, / Le temps dont on s'éprenne $\left.{ }^{25}\right)$, mais le non-sens, parce qu'il est cette possibilité toujours présente où l'expérience à tout moment peut s'effondrer, affaire de connaissance («Ce fut d'abord une étude. J'écrivais des silences, des nuits, je notais l'inexprimable. Je fixais des vertiges $»^{26}$ ). Aussi n'y a-t-il de sens que comme sens naissant, qui s'enlève sur fond d'une altérité (le danger) ou d'une antériorité (l'expérience comme totalité indifférenciée) dont la menace ou la présence ne pourront jamais être définitivement écartées.

\footnotetext{
${ }^{22}$ Jean-Yves Lacoste, Etre en danger, p. 369.

${ }^{23}$ Jean-Yves Lacoste, Recherches sur la parole, Louvain-La Neuve, Peeters, 2915, p. 211

${ }^{24}$ Jean-Yves Lacoste, Thèses sur le vrai, Paris, PUF, 2018, p. 188, 191 sq.

${ }^{25}$ Rimbaud, Une saison en enfer, « Alchimie du verbe »

${ }^{26}$ Ibid.
} 
Au commencement pour nous est le chaos, espace béant et sans direction, sans haut ni bas, sans avant ni arrière, sans orient, et où nous sommes jetés ${ }^{27}$. A moins que jetés dans un espace sans haut ni bas veuille dire : jetés en bas. A moins que là où il n'y a aucune différence pour s'orienter dans l'expérience - selon la différence du sens et du non-sens, du haut et du bas, de l'avant et de l'après - « le clair-obscur du monde » (Jean-Yves Lacoste) ne soit rendu à une obscurité plus grande que la clarté, et le tout de l'expérience à sa vanité ${ }^{28}$. L’absence de sens, le non-sens - pour l'existant, non pour le logicien, la frontière qui les sépare est quasi-imperceptible. «Au commencement est le chaos » - ce mot est du poète Hésiode, et il donne à penser. Mais ce commencement ouvre aussi une histoire - et quelle histoire puisqu'il s'agit de la Théogonie. Au premier mot succède un second puis un troisième, commencement d'une histoire où il est permis d'espérer.

Donc, avant tout, fut Abîme, puis Terre aux larges flancs, assise sûre à jamais offerte à tous les vivants, et Amour, le plus beau parmi les dieux immortels, celui qui rompt les membres et qui, dans la poitrine de tout dieu comme de tout homme, dompte le cœur et le sage vouloir. ${ }^{29}$

Au commencement est le tout indifférencié de l'expérience à même lequel il importe de tracer des différences. Avançons ici l'hypothèse que ces première différences sont esthétiques en ce qu'elles relèvent de l'espace et du temps. Ce qui revient à dire que le sens (compris comme signification) s'ancre dans le sens (compris comme sensation).

Dimension spatiale du sens. Sans doute n'y a-t-il pas de schème plus manifeste de la différence comme arrachement du sens au non-sens que ce mouvement qui prend son départ dans un point pour en rejoindre un autre - ce qui répond exactement à la troisième signification du mot sens, celle du sens comme direction, mais troisième manière d'en comprendre le concept qu'il faut penser dans son rapport aux deux autres (la signification, la sensation). Ce qu'Henri Maldiney a su parfaitement

${ }^{27}$ Tel est bien le sens premier du mot Khaos - celui d'Hésiode repris par Henri Maldiney : « La notion de chaos est en effet semblable à celle d'abîme, non venu du grec abyssos : sans fond, impénétrable, qui substantivé désigne le vide infini. Le chaos est une béance non seulement sans fond, mais sans aucune direction. Il n'y a en lui ni haut, ni bas, ni droite, ni gauche, ni avant, ni arrière, ni aucune système de coordonnées possibles. Il est hors dimension » (Avènement de l'œuvre, Saint-Maximin, Théétète-éditions, 1997, p. 77 sq). L'autre mot vers lequel se tourne Maldiney est celui d'Anaximandre cette fois, dans une sentence dont il faudrait suivre l'interprétation chez Heidegger, Maldiney et surtout Patocka.

${ }^{28}$ Cette leçon est aussi des Evangiles : à quoi bon amasser des biens si notre âme ignore pour quoi vivre? « Mais Dieu lui dit : "Insensé, cette nuit même, on va te redemander ton âme. Et ce que tu as amassé, qui l'aura ?" Ainsi en est-il de celui qui thésaurise pour lui-même, au lieu de s'enrichir en vue de Dieu » (Lc 12, 20-21).

${ }^{29}$ Hésiode, Théogonie, vers 116-122, trad. P. Mazon, Paros, Les Belles Lettres, 1928, p. 36. 


\section{JÉRÔME DE GRAMONT}

expliquer : « Les racines premières de la langue montrent comment le sens-direction sous-tend le sens-signification ${ }^{30} »$. Pour qu'une différence de sens puisse naître, il faut que l'existant s'arrache sur fond de l'antériorité d'un chaos ou de l'altérité d'un non-sens pour aller là-bas, vers un lieu qui lui serve d'orient. D'ici vers là-bas, du lieu où il est jeté vers cet autre lieu au-devant duquel il jette son regard, et le corps entier, et tout le poids de son existence. D'ici vers là-bas, d'une manière d'être au monde rivée à l'être-en bas, d'une manière d'être jeté qui l'écrase vers le bas, jusqu'à cette puissance d'ascension qui le fait exister au plus haut de soi parce qu'il se jette désormais vers le haut - en un mouvement de transcendance qui commence par la surrection du corps, celui-ci luttant contre sa propre pesanteur pour se ramasser tout entier dans la puissance de sa verticalité.

Même s'il convient de faire droit aux six dimensions fondamentales qui permettent au corps de se mouvoir (vers la gauche, vers la droite, en avant, en arrière, vers le haut et vers le bas), la primauté revient à la dimension de la verticalité parce que l'orientation vers le haut est sans variation et que l'être au sol donc en bas constitue le point-zéro de tout mouvement. Ma main gauche restera à jamais ma main gauche, mais ce qui se tient à ma gauche, à tout moment, par le plus simple mouvement du corps, peut passer à ma droite. De même, ce qui est en avant pour moi restera toujours l'en-avant du corps, mais il en va tout autrement de l'être an avant des choses qui à tout moment peut passer derrière mon dos. Alors que le ciel en sa hauteur restera toujours en haut et la terre en bas. Un ciel vers lequel se dresser en s'arrachant à la pesanteur de la terre. Aussi cette inscription dans l'espace d'un sens trouve-t-elle chez le Platon du Théétète sa parfaite description :

Mais il est impossible que le mal disparaisse, Théodore ; car il y aura toujours, nécessairement, un contraire du bien. Il est tout aussi impossible qu'il ait son siège parmi les dieux : c'est donc la nature mortelle et le lieu d'ici-bas que parcourt fatalement sa ronde. Cela montre quel effort s'impose : d'ici-bas vers là-haut s'évader au plus vite. ${ }^{31}$

Où il faut que le corps lutte contre soi, contre son évidente pesanteur, pour s'emparer de sa puissance de légèreté qui l'emporte vers le haut. D'une certaine manière, l'âme est le nom que Platon aura donné à cette puissance du corps de s'arracher à son propre poids $^{32}$. Où le haut et le bas, première dimension du sens, s'éprouve à travers

${ }^{30}$ Henri Maldiney, L'art, l'éclair de l'être, Seyssel, Editions Comp'Act, 1993, p. 349 (nouvelle édition Paris, Cerf, 2012, p. 292). Voir aussi p. 101/85, où après avoir exposé les trois sens du mot sens : sensdirection, sens-sensation et sens-signification, il poursuit : « Le sens-signification est issu des deux premiers, pour autant que ceux-ci concernent le rapport au monde d'un existant ».

${ }^{31}$ Platon, Théétète $176 \mathrm{a}$ - trad. A Diès (Paris, Les Belles Lettres, 1955).

$3^{2}$ D'où cette formule lumineuse d'Elsa Ballanfat à la suite d'une lecture du Phèdre où le fameux «mythe de l'âme » est reversé au compte du corps et de son pouvoir d'élévation - presque son 
l'antagonisme du lourd (qui nous retient vers le bas) et du léger (qui s'emporte vers le haut). Il faut donner raison au romancier Milan Kundera quand il écrit dans L'insoutenable légèreté de l'être: «Une seule chose est certaine. La contradiction lourd-léger est la plus mystérieuse et la plus ambiguë de toutes les contradictions ${ }^{33}$ », mais aussi corriger son affirmation, et voir dans cette contradiction la plus évidente épreuve par l'existant de l'antagonisme du sens et du non-sens.

Que le lourd puisse se retourner en léger, que le poids du corps et de l'existence entière, littéralement son fardeau, l'accablement de celui qui est jeté dans le monde et comme abandonné, et pour qui selon l'expression même de Heidegger au $§ 29$ de Etre et temps « l'être est devenu manifeste comme un poids $»^{34}$, puisse être suspendu pour que le pesant soit rendu à sa possible légèreté, voilà ce qui est digne de description et de pensée. Que s'ouvre l'espace des possibles, non plus seulement pour nous exposer au Danger, mais pour nous tourner vers la promesse d'une légèreté de l'existence conquise sur sa pesanteur, voilà ce dont il faut s'étonner, ce qu'il faut espérer et ce que la philosophie a pour tâche de comprendre. Voilà pour nous aujourd'hui la tâche, celle qu'ont vue en des pages sur lesquelles il faudrait s'arrêter longtemps Augustin, Kierkegaard ou Michel Henry ${ }^{35}$ :

'Le bagage que j'impose est léger'. Tout autre te pèse et t'écrase ; mais celui du Christ t'allège et relève ; tout autre pèse son poids, celui du Christ donne des ailes. ${ }^{36}$

pouvoir de danser : « L'âme est un nom posé sur une aspiration du corps » (La traversée du corps. Regard philosophique sur la danse, Paris, Hermann, 2015, p. 97).

${ }^{33}$ Milan Kundera, L’insoutenable légèreté de l'être, trad. F. Kerel revue par l'auteur, Paris, Gallimard, 1987, p. 14.

${ }^{34}$ « L'être est devenu manifeste comme un poids » (traduction E. Martineau, Paris, Authentica, 1985, p. 113) ; la traduction Boehm-Waelhens donne : "L'existence se manifeste comme un fardeau (Paris, Gallimard, 1964, p. 168). Jean Greisch commente brièvement : « la fait de se sentir "accablé" ne nous dit pas seulement quelque chose de nous-mêmes, mais de l'être. L'être a le pouvoir de nous accabler, de nous peser, de se révéler comme un fardeau » (Ontologie et temporalité, Paris, PUF, 1994, p. 178). Mais il n'y a rien là qu'Augustin n'ait déjà dit : "Je suis un poids pour moi-même " (Confessions $\mathrm{X}, 28,39$ ) et Heidegger relevé dans son cours du semestre d'été 1921 : «Il s'agit de cerner de manière plus précise ce caractère fondamental dans lequel Augustin expérimente la vie facticielle, c'est-à-dire la tentatio, et de comprendre à partir de là en quel sens celui qui vit dans une telle lucidité et à un tel niveau d'accomplissement est nécessairement un fardeau pour lui-même » (Phénoménologie de la vie religieuse, trad. J. Greisch, Paris, Gallimard, 2012, p. 230).

${ }^{35}$ Première esquisse de notre part dans « Le poids de la joie », publié dans Nunc n 18 , mars 2009 et repris dans Au commencement. Parole, Regard, Affect.

${ }^{36}$ Saint Augustin, Commentaire du Psaume 59,8 - cité in Jean-Luc Marion, Au lieu de soi, Paris, PUF, 2008 (voir son commentaire p. 212-216 et tout le $\S 41$, «Pondus meum », p. 352-365). Sur ce thème on lira avec profit Emmanuel Falque, «Le Haut Lieu du soi : une disputatio théologique et phénoménologique », Revue de métaphysique et de morale, juillet 2009, p. 372 sq, 375-379. 


\section{JÉRÔME DE GRAMONT}

Christ voulut enseigner ce qu'il montra par son exemple : le fardeau est léger, même quand la souffrance accable. Le fardeau reste ainsi en un sens le même, puisqu'il est la souffrance, la lourde souffrance ; et cependant il devient léger. Le lot de l'homme ici-bas n'a pas changé par suite de l'apparition du christianisme dans le monde. Un chrétien peut avoir à endurer exactement les mêmes souffrances que celles qu'a connues l'humanité avant la venue de Christ ; pour lui toutefois, de pesant, le fardeau devient léger. ${ }^{37}$

Or cette transformation du fardeau le plus lourd en ce qui est le plus léger, cette transsubstantiation magique elle aussi de la plus grande souffrance dans l'ivresse de l'amour sans limites, tout cela n'advient que chez celui en qui - à l'image du Christ - l'auto-affection de la Vie absolue s'est substituée à l'autoaffection du simple vivant donné à lui-même sans l'avoir voulu, dans l'autoaffection de cette Vie absolue et par elle cependant. Tout cela n'advient en lui que si, vivant sa condition de Fils et n'étant plus rien d'autre qu'elle, s'éprouvant soi-même dans l'épreuve de la vie infinie et vivant de cette épreuve, il est né une seconde fois re-généré dans la seconde vie. ${ }^{38}$

Dimension temporelle du sens. A l'évidence il était impossible de décrire la « direction de sens ${ }^{39}$ sans mêler l'espace et le temps. Si la différence d'espace entre haut et bas s'éprouve bien dans l'antagonisme entre pesanteur et légèreté, celui-ci est rendu manifeste à son tour par les mouvements adverses qui attirent le corps vers le bas ou l'emportent vers le haut. Encore faut-il qu'il y ait temps pour rendre possible ce passage d'une région vers l'autre, dans un mouvement du haut vers le bas (chute) ou du bas vers le haut (ascension). Où il y a direction il faut encore qu'il y ait succession. Lu dans l'horizon maintenant du temps, la direction de sens ne suppose pas seulement une différence mais une dissymétrie foncière entre un avant et un après, un point de départ et un point d'arrivée, un premier et un dernier mot. Où il y a sens, il ne suffira pas de constater une succession aléatoire où la joie vienne après la peine, et réciproquement, selon les hasards de ce qui nous arrive, mais il doit y avoir une direction, l'orient de cette différence, pour qu'un premier mot

\footnotetext{
${ }^{37}$ Kierkegaard, L'Evangile des souffrances (1847), trad. P.-H. Tisseau, Paris, Euvres complètes t. XIII, 1966, p. 266. Voir notre commentaire dans Le Discours de la vie, Paris, L'Harmattan, 2001, p. 216-229 (« Le retournement de la souffrance en joie »).

${ }^{38}$ Michel Henry, C'est moi la vérité, Paris, Seuil, 1996, p. 262. Sur le fardeau et la nouvelle naissance, comme sur la christologie henryenne qui rend possible ce paradoxe du lourd devenu léger, voir notre étude « De l'historial de la vie à l'histoire du vivant », in La vie et les vivants. (Re)-lire Michel Henry, Grégori Jean, Jean Leclercq et Nicolas Monseu éd., Presses Universitaires de Louvain, 2013.

39 Concept repris à Ludwig Binswanger, et à propos duquel Henri Maldiney peut écrire : «cette alliance de mots [rend] sensible l'unité du sens-direction et du sens-signification » (Regard Parole Espace, Lausanne, L’Age d'homme, 1973, p. 77 sq ; nouvelle édition Paris, Cerf, 2012, p. 143).
} 
nécessairement devienne un dernier mot, que la joie vienne toujours après la peine, ou que le lourd à tout moment puisse se transformer en légèreté.

Pour que naisse le sens, il ne suffira pas que du temps s'écoule mais il faudra que son passage, qui fait revenir un présent toujours vivant, soit porteur d'une puissance d'incessant renouvellement. Il ne faudrait pas surtout que le dernier mot, celui qui détient l'eschatologie du sens, revienne à la mort ou à la logique de l'être-vers-lamort, mais bien plutôt à cette puissance de commencer et de recommencer que pourrait traduire alors l'expression d'être-vers-la-naissance ${ }^{40}$. Aller vers sa légèreté (et vers le haut), aller vers sa naissance - ces deux mouvements vont de pair. Ce qu'Henri Maldiney, commentant Binswanger, a su dire :

« Si nous disons que le soir tombe et que le jour se lève, c'est à raison d'une liaison nonthématique, éprouvée entre l'éclairement, l'ascension (et la naissance) et l'assombrissement, la chute (et la mort).$^{41}{ }^{\prime}$

Reste que cette naissance du sens ne va pas sans paradoxe, puisque le corps doit s'arracher à sa propre pesanteur et au mouvement naturel de sa chute, et que le passage du temps semble conduire toute chose vers sa ruine et l'existence vers sa mort. Au renversement du lourd en léger correspond alors le retournement de la flèche du temps et la possibilité d'aller non pas vers notre mort mais vers notre puissance de commencer et de recommencer, vers notre naissance ou notre nouvelle naissance.

\footnotetext{
${ }^{40}$ Ce qu'aura bien montré la phénoménologie de Jean-Yves Lacoste : « Nous nous heurtons à la mort comme à la seule eschatologie qui ait une réalité ontique, et pouvons être tentés de l'interpréter comme eschatologie du sens. Nous disions que les enjeux du sens débordent en fait les conditions auxquelles, à l'ombre de la mort, nous avons du temps » (Note sur le temps, Paris, PUF, 1990, p. 201). Mais depuis l'événement de Pâques une autre logique se fait jour, qui est proprement une « logique du commencement » (p. 202).

${ }^{41}$ Henri Maldiney, Regard Parole Espace, p. 101/147.
} 